\title{
Interpreting oxygen vacancy migration mechanisms in oxides using the layered structure motif
}

\author{
Blas Pedro Uberuaga ${ }^{a}$, Kurt E. Sickafus ${ }^{b}$ \\ ${ }^{a}$ Materials Science and Technology Division, Los Alamos National Laboratory, Los Alamos, NM 87545 \\ ${ }^{b}$ Department of Materials Science and Engineering, University of Tennessee, Knoxville, TN
}

\section{Abstract}

The manner in which oxygen vacancies migrate in oxide ceramics is analyzed in terms of an atom layer stacking motif to elucidate similarities and differences in migration mechanisms as a function of crystal structure and chemistry. In particular, five oxide structures are examined: rocksalt, spinel, bixbyite, pyrochlore, and fluorite. While there are similarities that are related to structure in the types of migration mechanisms that are possible, there are significant differences as a consequence of chemistry and deviations from the parent structures (rocksalt and fluorite). One primary difference occurs because of structural relaxations related to the complex chemistry in spinel and pyrochlore in which oxygen ions tend to form localized groupings within which oxygen vacancy hopping mechanisms are particularly fast. While these mechanisms cannot lead to net migration, a kinetic Monte Carlo model does show that they do influence overall diffusivity. We conclude that the atom layer stacking motif is an effective scheme to understand the dependency of oxygen vacancy migration mechanisms on oxide crystal structure.

Keywords:

oxides, layered structure motif, defect kinetics, oxygen vacancy

\section{Introduction}

Oxide ceramics are a critical component of many technologies, from solid oxide fuel cells [1] to nuclear fuels and waste forms $[2,3]$ to dielectrics in semiconductor devices [4]. In many of these applications, the mobility of ionic defects, particularly anion defects, are key factors determining the suitability of the oxide for that application. For example, solid oxide fuel cells and fast ion conductors function precisely because of the mobility of anions within the lattice [5].

Atomistic simulations can provide important details regarding the properties of defects in these materials [6]. Such simulations have revealed that interstitial clusters in $\mathrm{MgO}$ and $\mathrm{UO}_{2}$ have high mobilities [7, 8], that cation Frenkel pair recombination in pyrochlores always leads to antisite formation $[9,10,11]$, and that, in $\mathrm{AB}_{2} \mathrm{O}_{4}$ spinel, $\mathrm{B}$ cation diffusion occurs via a vacancy mechanism on the $\mathrm{A}$ sublattice [12]. However, even the most sophisticated simulations must still be interpreted and placed into context for insight to be gained. This is particularly true in complex materials, where simply reporting mechanisms does not necessarily provide an understanding that is transferrable to other situations nor the generalizations that highlight commonalities and differences between, for example, related but different crystal structures.

Email address: blas@lanl.gov (Blas Pedro Uberuaga)
Here, we use atomistic simulations to examine the mechanisms responsible for doubly charged oxygen vacancy migration in a series of materials that vary in relative oxygento-metal content but are structurally related. In particular, we consider rocksalt and spinel, spinel being structurally related to rocksalt, and fluorite along with two fluorite-derivative structures, specifically bixbyite and pyrochlore. Upon irradiation, spinel can transform into a disordered rocksalt structure [13], while both bixbyite and pyrochlore transform into disordered fluorite structures $[14$, 15]. We interpret the migration mechanisms in terms of an atom layer stacking model recently developed by Sickafus et al. [16] By comparing the behavior of oxygen vacancies in these compounds, we elucidate similarities and differences in oxygen vacancy migration mechanisms that depend on the structure and chemistry of these compounds.

\section{Methodology}

We use temperature accelerated dynamics (TAD) [17] along with pair potentials to examine the migration mechanisms of oxygen vacancies in a variety of oxide compounds. The use of TAD eliminates any bias in assumed mechanisms, because a molecular dynamics (MD) trajectory is used to discover pathways dynamically. Once an event has been identified, the nudged elastic band (NEB) [18] method is used to characterize the event by finding the relevant saddle point. Depending on the compound, these 
simulations are performed with a low temperature of between 600 and $2000 \mathrm{~K}$ for simulation times ranging from nanoseconds to 0.1 milliseconds (no attempt was made to optimize parameters for a given system).

We chose to examine five different oxide structures rocksalt, spinel, fluorite, bixbyite, and pyrochlore - which vary in stoichiometry between rocksalt and fluorite as illustrated in Figure 1. Rocksalt and fluorite were chosen as they are the simplest compounds with integer oxygen/metal $(\mathrm{O} / \mathrm{M})$ ratios. The spinel structure is related to that of rocksalt while both bixbyite and pyrochlore are fluorite derivatives. Thus, choosing these compounds allows us to examine how the basic crystal structure of two families of compounds, as well as the complexities involved with deviations from integer stoichiometry, influence oxygen vacancy mobility. The specific chemical compounds simulated were $\mathrm{MgO}, \mathrm{MgAl}_{2} \mathrm{O}_{4}, \mathrm{CeO}_{2}, \mathrm{Er}_{2} \mathrm{O}_{3}$, and $\mathrm{Dy}_{2} \mathrm{Ti}_{2} \mathrm{O}_{7}$. The interatomic interactions were described with a standard Buckingham potential [19] with long-range Coulomb interactions calculated using Ewald summation [20]. The potential parameters for these different compounds were taken from the literature. For $\mathrm{MgO}$, we used the parameter set by Lewis and Catlow [21]. For $\mathrm{MgAl}_{2} \mathrm{O}_{4}$, we used the parameter set reported by Smith et al. [22]. We used the parameters of Vyas et al. [23] for $\mathrm{CeO}_{2}$ and those of Kittiratanawasin et al. [24] for $\mathrm{Er}_{2} \mathrm{O}_{3}$. Finally, for $\mathrm{Dy}_{2} \mathrm{Ti}_{2} \mathrm{O}_{7}$, we used the parameter set developed by Stanek et al. [25]. While we expect the barriers we report for various migration mechanisms to be dependent on the parameter set used, we also expect the basic physical trends to be well captured by these potentials. We note that these are fixed charge models and thus the vacancy always has the same charge state, formally +2 .

All simulations were conducted using periodic boundary conditions. The cell size was typically the minimum possible size satisfying the minimum-image convention for a potential cutoff of $8 \AA$. This lead to simulation sizes of 511 atoms for rocksalt, 447 atoms for spinel, 639 atoms for bixbyite, 703 atoms for pyrochlore, and 767 atoms for fluorite. These numbers account for the missing atom resulting from the creation of the oxygen vacancy.

Many of the results reported here are based on previous simulations in which, typically, we provided the value for the energy barrier of the rate limiting process for migration of the oxygen vacancy in the given compound, but did not elaborate any further on the actual pathways or alternative mechanisms. In particular, we reported migration barriers for the oxygen vacancy in $\mathrm{MgO}$ [26], spinel [27], $\mathrm{Er}_{2} \mathrm{O}_{3}$ [24], and pyrochlore [28]. Further, the behavior of the oxygen vacancy in $\mathrm{Er}_{2} \mathrm{O}_{3}$ was examined in detail by Kittiratanawasin and Smith [29].

\section{Layered Motif Structure of Oxides}

The structure of oxides, particularly complex oxides containing more than 2 species, can be analyzed in a variety of ways. One common approach is to visualize the

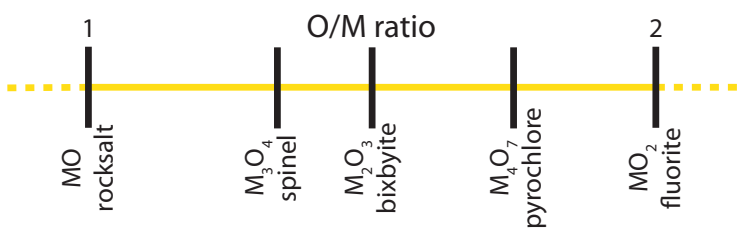

Figure 1: Relationship between the five oxide compounds examined along an oxidation line scaled by relative oxygen/metal ratio.

polyhedra that comprise the structure [30]. Topology has also been a powerful way to understand the structure of complex oxides [31]. More recently, Sickafus and colleagues have examined the structure of oxides from the point of view of an atom layer stacking model [16], building upon concepts originally proposed by Iida [32]. In this view of oxides, the atomic structure of a given compound is visualized as a series of atomic planes stacked in a [111] direction (for cubic compounds). Each of these planes exhibit particular arrangements of atoms that generalize across a wide range of oxide structures. These stacking motifs, illustrated in Figure 2 for the compounds studied here, form the basis of each compound. It is simply the order in which they are stacked that governs the structure of the oxide.

The basic motif for rocksalt-based compounds (rocksalt and spinel) is alternating layers of metal (M) and oxygen (O). For fluorite and fluorite derivatives such as bixbyite and pyrochlore, the motif is O-M-O. The specific stacking sequences for rocksalt, spinel, bixbyite, pyrochlore, and fluorite are given in Table 1. In this view, the differences in structure between fluorite, pyrochlore and bixbyite are related to the individual structure of each layer. For example, in fluorite, all of the oxygen and metal layers are comprised of fully dense hexanets as illustrated in Figure 2. In bixbyite, in contrast, the oxygen layers are not fully dense, but rather contain structural vacancies that accommodate the relative difference in stoichiometry between bixbyite and fluorite. In the case of bixbyite, these vacancies order in a very well defined pattern, referred to as a "wishbone kagome" structure (Figure 2). Similarly, pyrochlore differs from fluorite in that half of the oxygen planes contain structural vacancies that form a "kagome" pattern. Pyrochlore has the extra complexity of having two different cationic species. These form fully dense planes of cations, but these planes alternate in chemistry, with some forming a kagome lattice of A cations with B cations filling the central hole of the kagome pattern, and others reversing the role of A and B cations (referred to as a "filled kagome pattern" in Figure 2). Finally, spinel is related to rocksalt in that all of the oxygen layers are fully dense, but the metal layers alternate between a kagome pattern of $B$ cations and a set of three quarter-dense planes of B-A-B cations, which together form a $\frac{3}{4}$ dense hexanet. 


\begin{tabular}{c|c|c|c|c|c}
$\begin{array}{c}\text { Fraction } \\
\text { along } \\
{[111]}\end{array}$ & $\mathrm{MO}$ & $\mathrm{M}_{3} \mathrm{O}_{4}$ & $\mathrm{M}_{2} \mathrm{O}_{3}$ & $\mathrm{M}_{4} \mathrm{O}_{7}$ & $\mathrm{MO}_{2}$ \\
$\left(\mathrm{AB}_{2} \mathrm{O}_{4}\right)$ & & $\left.\mathrm{A}_{2} \mathrm{~B}_{2} \mathrm{O}_{7}\right)$ & \\
\hline $23 / 24$ & & & & & \\
$21 / 24$ & & $\mathrm{~B}_{k}$ & & $\mathrm{O}_{f d}$ & \\
$20 / 24$ & $\mathrm{M}_{f d}$ & $\mathrm{O}_{f d}$ & $\mathrm{O}_{w k}$ & $\mathrm{~A}_{k} \mathrm{~B}$ & \\
$19 / 24$ & & $\mathrm{O}_{f d}$ & $\mathrm{O}_{f d}$ \\
$18 / 24$ & & $\mathrm{~B}_{q d}$ & $\mathrm{M}_{f d}$ & $\mathrm{O}_{k}$ & $\mathrm{M}_{f d}$ \\
$17 / 24$ & & $\mathrm{~A}_{q d}$ & & $\mathrm{~B}_{k} \mathrm{~A}$ & \\
$16 / 24$ & $\mathrm{O}_{f d}$ & $\mathrm{O}_{f d}$ & $\mathrm{O}_{w k}$ & $\mathrm{O}_{k}$ & $\mathrm{O}_{f d}$ \\
$15 / 24$ & & & & & \\
$14 / 24$ & & $\mathrm{~B}_{k}$ & & $\mathrm{O}_{f d}$ & \\
$13 / 24$ & & & & $\mathrm{~A}_{k} \mathrm{~B}$ & \\
$12 / 24$ & $\mathrm{M}_{f d}$ & $\mathrm{O}_{f d}$ & $\mathrm{O}_{w k}$ & $\mathrm{O}_{f d}$ & $\mathrm{O}_{f d}$ \\
$11 / 24$ & & $\mathrm{~A}_{q d}$ & & & \\
$10 / 24$ & & $\mathrm{~B}_{q d}$ & $\mathrm{M}_{f d}$ & $\mathrm{O}_{k}$ & $\mathrm{M}_{f d}$ \\
$9 / 24$ & & $\mathrm{~A}_{q d}$ & & $\mathrm{~B}_{k} \mathrm{~A}$ & \\
$8 / 24$ & $\mathrm{O}_{f d}$ & $\mathrm{O}_{f d}$ & $\mathrm{O}_{w k}$ & $\mathrm{O}_{k}$ & $\mathrm{O}_{f d}$ \\
$7 / 24$ & & & & & \\
$6 / 24$ & & $\mathrm{~B}_{k}$ & & $\mathrm{O}_{f d}$ & \\
$5 / 24$ & & & & $\mathrm{~A}_{k} \mathrm{~B}$ & \\
$4 / 24$ & $\mathrm{M}_{f d}$ & $\mathrm{O}_{f d}$ & $\mathrm{O}_{w k}$ & $\mathrm{O}_{f d}$ & $\mathrm{O}_{f d}$ \\
$3 / 24$ & & $\mathrm{~A}_{q d}$ & & & \\
$2 / 24$ & & $\mathrm{~B}_{q d}$ & $\mathrm{M}_{f d}$ & $\mathrm{O}_{k}$ & $\mathrm{M}_{f d}$ \\
$1 / 24$ & & $\mathrm{~A}_{q d}$ & & $\mathrm{~B}_{k} \mathrm{~A}$ & \\
$0 / 24$ & $\mathrm{O}_{f d}$ & $\mathrm{O}_{f d}$ & $\mathrm{O}_{w k}$ & $\mathrm{O}_{k}$ & $\mathrm{O}_{f d}$ \\
\hline & $\mathrm{MO}$ & $\mathrm{M}_{3} \mathrm{O}_{4}$ & $\mathrm{M}_{2} \mathrm{O}_{3}$ & $\mathrm{M}_{4} \mathrm{O}_{7}$ & $\mathrm{MO}_{2}$
\end{tabular}

Table 1: Stacking motifs, in terms of the layer patterns in Figure 2 , for rocksalt $(\mathrm{MO})$, spinel $\left(\mathrm{M}_{3} \mathrm{O}_{4}\right)$, bixbyite $\left(\mathrm{M}_{2} \mathrm{O}_{3}\right)$, pyrochlore $\left(\mathrm{M}_{4} \mathrm{O}_{7}\right)$, and fluorite $\left(\mathrm{MO}_{2}\right)$. The terminology used is: $\mathrm{O}_{f d}$ and $\mathrm{M}_{f d}$ indicate fully dense hexanets of oxygen and metal ions, respectively; $\mathrm{A}_{q d}$ and $\mathrm{B}_{q d}$ indicate quarter dense hexanets of $\mathrm{A}$ and $\mathrm{B}$ cations, respectively; $\mathrm{O}_{k}$ and $\mathrm{B}_{k}$ indicate kagome patterns of oxygen and $\mathrm{B}$ cations, respectively; $\mathrm{O}_{w k}$ indicates a wishbone kagome pattern of oxygen; and $\mathrm{B}_{k} \mathrm{~A}$ and $\mathrm{A}_{k} \mathrm{~B}$ indicate filled kagome patterns in which $\mathrm{B}$ forms a kagome pattern and $\mathrm{A}$ resides within the empty spaces of the kagome pattern and vice versa. The unit cells of spinel, bixbyite, and pyrochlore are double those of the parent rocksalt and fluorite structures. The table only indicates the layering of the various structural motifs represented in Figure 2. The real structures also include registry shifts to ensure the proper stacking sequence.
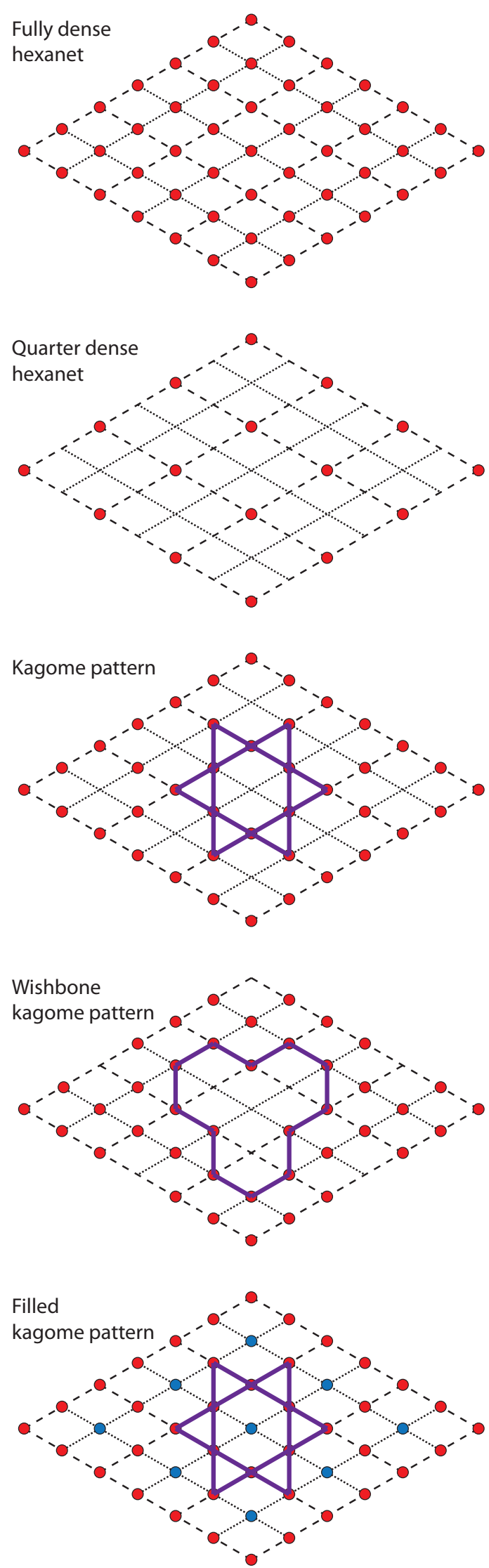

Figure 2: Idealized layer patterns that form the foundation of the structures of rocksalt, fluorite, pyrochlore, bixbyite, and spinel. These idealizations do not include the ionic distortions that occur 3 in the real compounds. 


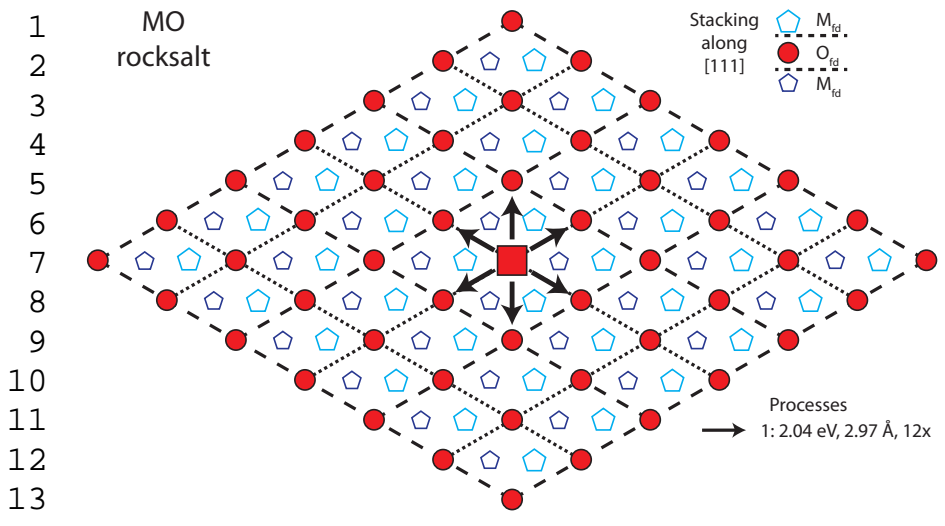

14

15 shown in Figure 4. The basic behavior is essentially the same for spinel and rocksalt: the oxygen vacancy can execute hops to the nearest neighbor oxygen ions within the $\{111\}$ planes. However, because of the structural relaxations described above, a consequence of the relative difference in stoichiometry between spinel and rocksalt and the structural cation vacancies that accommodate that difference, the 6 hops are not equivalent. Rather, two have a barrier of $1.50 \mathrm{eV}$, an additional two have a barrier of 1.66 $\mathrm{eV}$, and the other two processes that occur in rocksalt were never observed in the TAD simulations, even though the MD used to sample the pathways was done at a temperature of $3000 \mathrm{~K}$. The cation deficiency results in oxygen ions distorting away from the ideal structure such that, within a given (111) plane, oxygen ions tend to form structural units of three anions more tightly bound and thus further away from the other anions within the plane. This leads to three different anion-anion distances within a given plane (2.46, 2.88, and $3.27 \AA$ ), which correlate with the different magnitudes of the barriers. Thus, while the fully dense triangular lattice of the oxygen ions does provide the basic migration mechanisms of the oxygen vacancy, the details depend on the structural relaxations related to the chemistry of spinel.

When one considers the other $\{111\}$ planes that intersect the position of the vacancy, there are a total of 3 of the fastest jumps, 6 of the intermediate jumps, and 3 of the "forbidden" processes. If all things were equal, the fact that there are only 9 active pathways in spinel as compared to rocksalt would mean a reduction in rate of $9 / 12$ for oxygen vacancy migration. However, the barriers are also lower in spinel, meaning that oxygen vacancy migration is significantly faster in spinel than rocksalt. Further, the lower barrier process alone cannot lead to net diffusion. This is because this process simply cycles the vacancy between three connected sites within the plane and cannot take the vacancy further. Net diffusion requires long range migration of the vacancy, which is not possible via the smaller barrier process. The intermediate barrier process, with a degeneracy of 6 , is required for net diffusion of the oxygen vacancy. Finally, the magnitude of these barriers is very sensitive to chemistry.

Comparing the overall behavior between rocksalt and spinel, the dominant migration mechanisms for both structures occur within a given (111) plane. However, two of the mechanisms that are active in rocksalt are inactive in spinel, processes which take the oxygen vacancy near an A cation. In this system, the extra structural complexity of spinel reduces the number of pathways available for the oxygen vacancy.

\subsection{Fluorite, Bixbyite, and Pyrochlore}

\subsubsection{Fluorite}

Starting from the other end of the oxidation line (Figure 1), we next examine the behavior of oxygen vacancies in the fluorite structure. Figure 5 shows a schematic of 


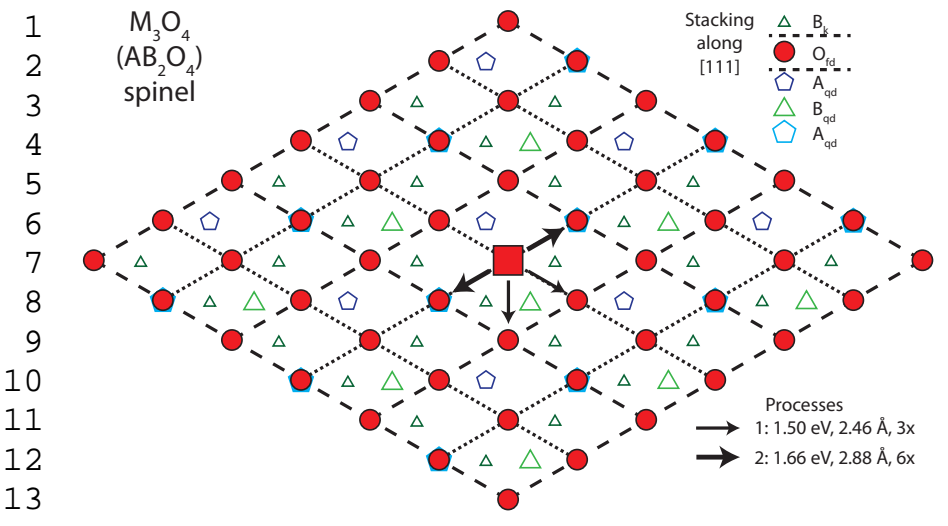

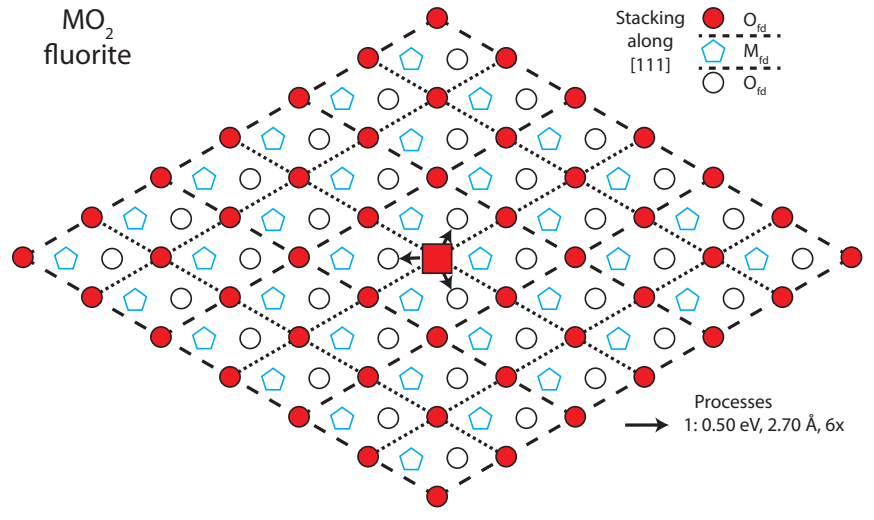

Figure 5: Schematic of the ideal structure of $\mathrm{MO}_{2}$ fluorite viewed down the (111) axis. The symbols are defined in the legend in the upper right while the migration mechanisms for the oxygen vacancy (the large filled square) are indicated by the arrows. In the legend in the lower right, the barrier for the process, the hop length (in the relaxed structure of the material), and the degeneracy of the mechanism are given as well as an arrow to identify the processes in the schematic. The different layers in the stacking sequence are distinguished by the dashed lines in the legend.

migrate within bixbyite. Two of these pathways are analogous to those exhibited in fluorite in which the vacancy hops along a [100] direction out of the current (111) plane and into the adjacent (111) plane. These have barriers of $1.01 \mathrm{eV}$ and $1.37 \mathrm{eV}$ for the bixbyite $\mathrm{Er}_{2} \mathrm{O}_{3}$. As opposed to fluorite, in which three such hops were possible, only two are possible in bixbyite as there is a structural vacancy in the adjacent (111) plane in the third direction. Further, it is possible for the oxygen vacancy to execute jumps that keep it within the original (111) plane, and such a mechanism has the lowest barrier. With a barrier of $0.81 \mathrm{eV}$, the vacancy can hop from the edge of one wishbone kagome unit to a neighboring one. Another move within the original (111) plane is also possible, which keeps the vacancy within the original wishbone kagome pattern, but it has a much higher barrier of $1.94 \mathrm{eV}$.

The smallest barrier process $(0.81 \mathrm{eV})$ does not lead to net diffusion. It simply takes the vacancy back and forth from the edge of one wishbone kagome unit to the neighboring one, leading to an oscillation of the vacancy between the two sites. For net diffusion, the second largest barrier process $(1.01 \mathrm{eV})$, which takes the vacancy from one (111) oxygen plane to the neighboring one, is required.

\subsubsection{Pyrochlore}

Finally, we examine the migration mechanisms of oxygen vacancies within the pyrochlore structure. The mechanisms found via TAD simulations are shown in Figure 7. The oxygen vacancy in pyrochlore exhibits behavior that is in some sense similar to bixbyite, but with extra complexity. First, as opposed to the other oxides we have considered here, in pyrochlore there are two inequivalent oxygen ions within the structure and the oxygen vacancy has a strong preference to reside in one site over the other 


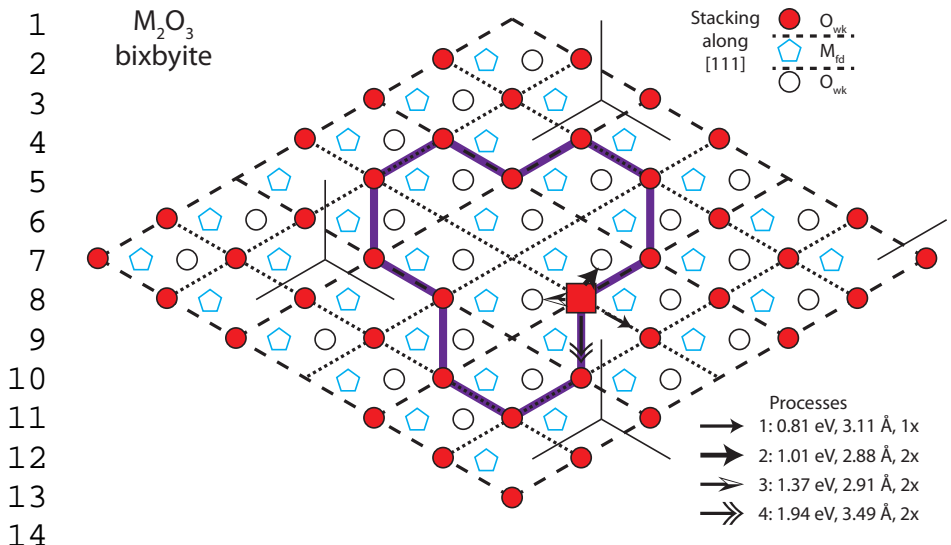

15

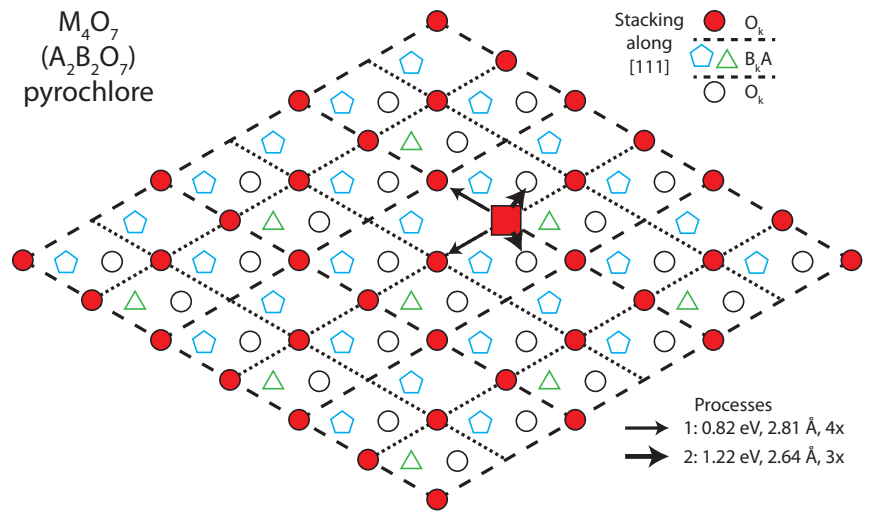

Figure 7: Schematic of the ideal structure of $\mathrm{M}_{4} \mathrm{O}_{7}$ pyrochlore viewed down the (111) axis. The symbols are defined in the legend in the upper right while the migration mechanisms for the oxygen vacancy (the large filled square) are indicated by the arrows. In the legend in the lower right, the barrier for the process, the hop length (in the relaxed structure of the material), and the degeneracy of the mechanism are given as well as an arrow to identify the processes in the schematic. The different layers in the stacking sequence are distinguished by the dashed lines in the legend.

within the initial (111) plane. This leads to an overall increase in the number of available pathways and, if all else were equal and all paths could lead to net migration of the vacancy, oxygen vacancy mobility would be higher in bixbyite and pyrochlore than in fluorite, in contrast to the relationship between spinel and rocksalt.

\subsection{Role of Chemistry}

Not only does the structure of the oxide influence the mobility of defects, but so does the chemistry. We have examined the mobility of the oxygen vacancy within a number of structurally identical but chemical different compounds to elucidate the role of chemistry on migration. The results are presented in Table 2. (Note that while $\mathrm{La}_{2} \mathrm{O}_{3}$ is typically found in a hexagonal phase, in thin film geometries it can adopt a bixbyite structure [35].) In the case of the bixbyite compounds, there is very little sensitivity between the barriers for the various processes and the chemistry of the compound, even though the chemistry varies significantly, with atomic numbers of $21(\mathrm{Sc})$, 57 (La), and 68 (Er).

In the case of pyrochlore, the barrier of the rate limiting process (Process 2) is very similar between the three chemistries examined. Thus, one would expect oxygen vacancy mobility to be very similar in these three compounds. However, there are significant differences in the energy of Process 1, which cycles the vacancy between a subset of sites within the anion sublattice. As the radius of the A cation decreases, so too does the barrier for Process 1. As mentioned above, Process 1 alone cannot lead to net migration of the vacancy - Process 2 is required. However, the barrier for Process 1 can influence the overall mobility of the defect, as discussed in the next section. 


\begin{tabular}{|c|c|c|c|c|}
\hline 1 & Process & \multicolumn{3}{|c|}{ Compound Chemistry } \\
\hline $\begin{array}{l}+ \\
2\end{array}$ & \multicolumn{4}{|c|}{ Spinel } \\
\hline 3 & & $\mathrm{MgAl}_{2} \mathrm{O}_{4}$ & $\mathrm{MgGa}_{2} \mathrm{O}_{4}$ & $\mathrm{MgIn}_{2} \mathrm{O}_{4}$ \\
\hline 4 & Process 1 & 1.50 & 1.47 & 0.29 \\
\hline 5 & Process 2 & 1.66 & 2.00 & 1.83 \\
\hline 6 & \multicolumn{4}{|c|}{ Bixbyite } \\
\hline 7 & & $\mathrm{Er}_{2} \mathrm{O}_{3}$ & $\mathrm{La}_{2} \mathrm{O}_{3}$ & $\mathrm{Sc}_{2} \mathrm{O}_{3}$ \\
\hline 8 & Process 1 & 0.81 & 0.84 & 0.81 \\
\hline 9 & Process 2 & 1.01 & 1.08 & 1.01 \\
\hline 0 & Process 3 & 1.37 & 1.42 & 1.38 \\
\hline[ & Process 4 & 1.94 & 1.89 & 2.07 \\
\hline 12 & \multicolumn{4}{|c|}{ Pyrochlore } \\
\hline 13 & & $\mathrm{Dy}_{2} \mathrm{Ti}_{2} \mathrm{O}_{7}$ & $\mathrm{Er}_{2} \mathrm{Ti}_{2} \mathrm{O}_{7}$ & $\mathrm{Lu}_{2} \mathrm{Ti}_{2} \mathrm{O}_{7}$ \\
\hline & Proce & 0.82 & 0.50 & 0.29 \\
\hline 15 & Process 2 & 1.22 & 1.21 & 1.20 \\
\hline
\end{tabular}

past experience $[7,26,39]$ indicates that, in particular for migration energies, the types of potentials used here are highly accurate when compared with experiment and density functional theory (DFT).

In $\mathrm{MgO}$, experiments examining interstitial loop growth rates have identified that oxygen vacancy migration has a barrier of about $1.9 \mathrm{eV}$ [40]. This agrees very well with our value of $2.04 \mathrm{eV}$. A similar study reported similar values $(2.0 \pm 0.7 \mathrm{eV})$ for the rate controlling (slowest) vacancy species in $\mathrm{MgAl}_{2} \mathrm{O}_{4}$ [41], though our previous calculations suggest this species is the $\mathrm{Al}$ vacancy [27]. To the best of our knowledge, there are no reported values for oxygen vacancy migration in spinels. Wirtz and Takiar examined oxygen diffusion in $\mathrm{In}_{2} \mathrm{O}_{3}$, which has the bixbyite structure [42]. They found a value of $1.4 \mathrm{eV}$, which is in reasonable agreement with DFT calculations for the doubly charged vacancy of $1.1 \mathrm{eV}$ [43]. Our value of $1.01 \mathrm{eV}$ for $\mathrm{Er}_{2} \mathrm{O}_{3}$ is slightly lower, though the chemistry is different and that may lead to some discrepancy (oxygen vacancy migration energies have not been reported for $\mathrm{Er}_{2} \mathrm{O}_{3}$ ). However, the experimental value agrees very well with the value we predict for process 3 , which may mean that at elevated temperatures, this process begins to contribute significantly. Indeed, kinetic Monte Carlo simulations do show an increase in the effective migration energy of the oxygen vacancy with temperature [43]. As discussed in Ref. [28], there is excellent agreement between the values for oxygen migration predicted by these potentials and experiment. Finally, in the case of $\mathrm{CeO}_{2}$, our value of $0.5 \mathrm{eV}$ for the migration energy of oxygen vacancies is in excellent agreement with both experiment (0.52 eV, Ref. [44]) and DFT (0.53 eV, Ref. [45]; $0.46 \mathrm{eV}$, Ref. [46]).

This discussion shows that, while the potentials used here and in numerous other studies do not provide a fully accurate description of the materials in question, they

\subsection{KMC simulations of vacancy mobility}

As discussed above, there are two different sets of processes in both spinel and pyrochlore, one that cycles the vacancy between a small connected set of sites and another that is required for net diffusion. While this second event is the rate limiting step for diffusion, the barrier of the first process, which depends strongly on the chemistry of either the spinel or pyrochlore (Table 2), will influence the overall diffusivity of the vacancy. To determine exactly what role Process 1 has on the mobility of the vacancy, we constructed a simple kinetic Monte Carlo (KMC) [47] model of a single hexanet layer in which the energy of placing a vacancy is the same at all sites, but the barriers between the sites differ in much the same way as observed in spinel (though all six vacancy hops are possible in this model, while only 4 actually occur in spinel). The model is illustrated in Fig. 8a. Thus, in the model, there are two processes, one process that only moves the vacancy amongst three nearest neighbor sites (Process 1) and a second process that is the rate limiting step for migration of the vacancy (Process 2). The mobility of the oxygen 
vacancy was then calculated as a function of the barrier for Process 1, which was varied between $0.5 \mathrm{eV}$ and $1.5 \mathrm{eV}$, and temperature, which was varied from $750 \mathrm{~K}$ to $10,000 \mathrm{~K}$ (this extremely high and unphysical temperature was used to identify the limiting trend in the behavior of the mobility); the barrier for Process 2 was held constant at 1 $\mathrm{eV}$.

The results of the KMC simulations are shown in Fig. 8b. First, at low temperature, as the barrier for Process 1 is reduced, the diffusivity of the vacancy, relative to a case were the barriers for both Process 1 and Process 2 are $1.0 \mathrm{eV}$, increases. The increase in diffusivity has a slight dependence on temperature, but for temperatures of $2000 \mathrm{~K}$ or less, tends towards 1.6-1.7. This increase is a consequence of the fact that, while the rate limiting step for diffusion still has a barrier of $1.0 \mathrm{eV}$ (Process 2 is the rate limiting step) an increase in the rate of Process 1 means that the vacancy can explore a sub-set of the lattice faster than it otherwise could. This accelerates the ability to jump to sites via Process 2 that are further away. In contrast, as the barrier for Process 1 becomes greater than Process 2 (a situation not observed in any of the oxides described above, but examined for completeness), the relative diffusivity of the vacancy decreases. This is because some pathways that were active when the barrier for Process 1 was smaller are now blocked and the number of pathways to diffuse through the system is reduced. The reduction in the relative diffusivity in this case is about a factor of 0.6 for temperatures of $2000 \mathrm{~K}$ or below. If the temperature is high enough (though unphysically so), the relative mobility of the vacancy does not depend on the barrier for Process 1 , as indicated by the $\mathrm{T}=10,000 \mathrm{~K}$ curve. This is because, at high enough temperature, all of the rates tend towards the same value and there is no dependence on the differences in barriers between the two processes.

A change in diffusivity by a factor of $0.6-1.7$ is rather modest and would likely be dwarfed by other factors, such as the amount of cation disorder in the system, the size of the preexponential factors (assumed here to all be $10^{13} / \mathrm{s}$ ) and anharmonicities [29], the charge state of the vacancy, or even impurities present in the material. However, the fact that there is a dependence in the mobility of the vacancy on the barrier for Process 1 shows that the migration of defects through these complex oxides is not trivial and that the rate limiting process alone is not enough to fully characterize the mobility of a given defect.

\section{Discussion and Conclusions}

As mentioned, in real complex oxides, a number of other factors certainly influence the mobility of oxygen vacancies within the lattice. In particular, both spinels and pyrochlores exhibit varying degrees of cationic disorder either naturally or induced by irradiation. This disorder will certainly influence oxygen vacancy kinetics. Interestingly, in spinels, this reduces the overall mobility of oxygen vacancies [27] while in pyrochlores disorder increases their

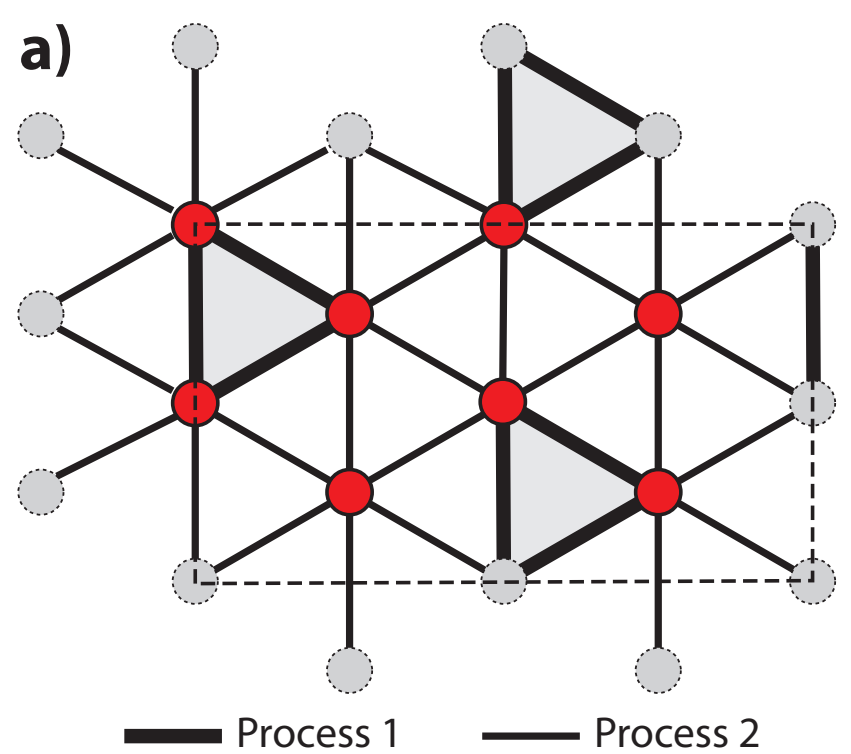

b)

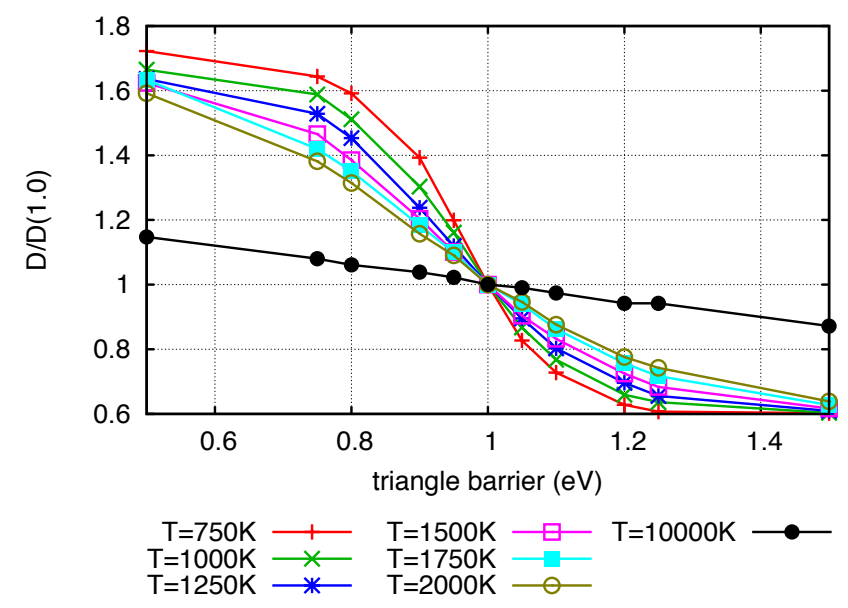

Figure 8: (a) Schematic of the KMC model of one (111) plane on the anion sublattice, or hexanet layer, typical of spinel or pyrochlore. The thick lines represent sites connected by Process 1 (typically the faster process) while thin lines represent connections via Process 2 . The dashed line indicates the orthonormal repeat cell of the layer. Red circles are sites within the unit cell while gray circles are sites outside the unit cell, shown for clarity. (b) The diffusivity of an oxygen vacancy as a function of the barrier for Process 1 , relative to the diffusivity when that barrier is $1.0 \mathrm{eV}$ (the same as for Process 2) for several temperatures. 
mobility [48]. Thus, the descriptions above provide a base to not only interpret mobility as a function of structure and chemistry, but could also be used to understand how antisite disorder influences defect mobility. Further, it is well established that the charge state of the defect, not accounted for in these simulations in which the oxygen vacancy always has a charge of +2 , modifies the defect mobility. Finally, in some materials the structure of the oxygen vacancy is more complex than for the compounds examined here. In some pyrochlores, for example, the oxygen vacancy forms a split structure [28]. Interpetting the pathways of more complicated defect structures in terms of the atom layer motifs used here may prove more difficult.

To conclude, we have examined the mobility of oxygen vacancies in a range of oxide crystal structures, interpretting that mobility in terms of a layered stacking motif. Using the stacking motif aids in highlighting both commonalities and differences in the oxygen vacancy migration mechanisms in these complex structures. Rocksaltstructured compounds (rocksalt and spinel) exhibit migration pathways for the oxygen vacancy that are dominated by mechanisms that are within the 111 planes, while those in fluorite-related compounds (fluorite, bixbyite, and pyrochlore) are dominated by inter-planar mechanisms (mechanisms that are not contained within a (111) plane). We find that the complex chemistries presented by spinel and pyrochlore change the mechanisms available to the vacancy, enhancing the number of pathways in pyrochlore as compared to fluorite but decreasing them in spinel as compared to rocksalt. The layered stacking motif provides new perspectives into the relationship between crystal structure and defect kinetics.

\section{Acknowledgements}

This work was supported by the U.S. Department of Energy, Office of Science, Basic Energy Sciences, Materials Sciences and Engineering Division. Los Alamos National Laboratory is operated by Los Alamos National Security, LLC, for the National Nuclear Security Administration of the (U.S.) Department of Energy under contract DEAC52-06NA25396. We thank Arthur F. Voter for helpful discussions.

\section{References}

[1] S. Hull, Rep. Prog. Phys. 67 (2004) 1233.

[2] D. Olander, J. Nucl. Mater. 389 (2009) 1.

[3] W. J. Weber, C. R. A. Catlow, L. W. Hobbs, H. Matzke, M. Nastasi, E. R. Vance, J. Mater. Res. 13 (1998) 1434.

[4] G. D. Wilk, R. M. Wallace, J. M. Anthony, J. Appl. Phys. 89 (2001) 5243.

[5] B. C. H. Steele, Mat. Sci. Eng. B13 (1992) 79.

[6] B. P. Uberuaga, D. A. Andersson, C. R. Stanek, Current Opinion in Solid State and Materials Science 17 (2013) 249.

[7] B. P. Uberuaga, R. Smith, A. R. Cleave, F. Montalenti, G. Henkelman, R. W. Grimes, A. F. Voter, K. E. Sickafus, Phys. Rev. Lett. 92 (2004) 115505.
[8] D. A. Andersson, F. J. Espinosa-Faller, B. P. Uberuaga, S. D. Conradson, J. Chem. Phys. 136 (2012) 234702.

[9] Y. H. Li, B. P. Uberuaga, C. Jiang, S. Choudhury, J. A. Valdez, M. K. Patel, J. Won, Y. Q. Wang, M. Tang, D. J. Safarik, D. D. Byler, K. J. McClellan, I. O. Usov, T. Hartmann, G. Baldinozzi, K. E. Sickafus, Phys. Rev. Lett. 108 (2012) 195504.

[10] R. Devanathan, W. J. Weber, J. D. Gale, Energy and Environ. Sci. 3 (2010) 1551.

[11] A. Chartier, G. Catillon, J. P. Crocombette, Phys. Rev. Lett. 102 (2009) 155503.

[12] S. T. Murphy, B. P. Uberuaga, J. B. Ball, A. R. Cleave, K. E. Sickafus, R. Smith, R. W. Grimes, Solid State Ionics 180 (2009) 1.

[13] M. Ishimaru, Y. Hirotsu, I. V. Afanasyev-Charkin, K. E. Sickafus, J. Phys.: Condens. Matter 14 (2002) 1237.

[14] M. Tang, J. A. Valdez, K. E. Sickafus, P. Lu, Appl. Phys. Lett. 90 (2007) 151907

[15] S. X. Wang, L. M. Wang, R. C. Ewing, K. V. G. Kutty, Mater. Res. Soc. Symp. Proc. 540 (1999) 355

[16] K. E. Sickafus, R. W. Grimes, S. M. Corish, A. R. Cleave, C. R. Stanek, B. P. Uberuaga, J. A. Valdez, Los Alamos Series Report LA-14205.

[17] M. R. Sorensen, A. F. Voter, J. Chem. Phys. 112 (2000) 9599.

[18] G. Henkelman, B. P. Uberuaga, H. Jónsson, J. Chem. Phys. 113 (2000) 9901.

[19] R. A. Buckingham, Proceedings of the Royal Society of London. Series A, Mathematical and Physical Sciences 168 (1938) 264.

[20] P. Ewald, Ann. Phys. 369 (1921) 253.

[21] G. V. Lewis, C. R. A. Catlow, J. Phys. C: Solid State Phys. 18 (1985) 1149.

[22] R. Smith, D. Bacorisen, B. P. Uberuaga, K. E. Sickafus, J. A. Ball, R. W. Grimes, J. Phys.: Condens. Matter 17 (2005) 875.

[23] S. Vyas, R. W. Grimes, D. H. Gay, A. L. Rohl, J. Chem. Soc., Faraday Trans. 94 (1998) 427.

[24] L. Kittiratanawasin, R. Smith, B. P. Uberuaga, K. E. Sickafus, A. R. Cleave, R. W. Grimes, Nucl. Instrum. Methods Phys. Res., Sect. B 266 (2008) 2691.

[25] C. R. Stanek, L. Minervini, R. W. Grimes, J. Am. Ceram. Soc. 85 (2002) 2792.

[26] B. P. Uberuaga, R. Smith, A. R. Cleave, G. Henkelman, R. W. Grimes, A. F. Voter, K. E. Sickafus, Phys. Rev. B 71 (2005) 104102.

[27] B. P. Uberuaga, D. Bacorisen, R. Smith, J. A. Ball, R. W. Grimes, A. F. Voter, K. E. Sickafus, Phys. Rev. B 75 (2007) 104116.

[28] M. Pirzada, R. W. Grimes, L. Minervini, J. F. Maguire, K. E. Sickafus, Solid State Ionics 140 (2001) 201.

[29] L. Kittiratanawasin, R. Smith, Nucl. Instrum. Methods Phys. Res., Sect. B 269 (2011) 1712.

[30] L. Pauling, The Nature of the Chemical Bond, Cornell University Press, Ithaca, New York, 1960.

[31] L. W. Hobbs, Nucl. Instrum. Methods Phys. Res., Sect. B 91 (1994) 30.

[32] S. Iida, J. Phys. Soc. Japan 12 (1957) 222

[33] K. E. Sickafus, J. M. Wills, N. W. Grimes, J. Am. Ceram. Soc. 82 (1999) 3279

[34] M. A. Subramanian, G. Aravamudan, G. V. S. Rao, Prog. Solid St. Chem. 15 (1983) 55.

[35] J. S. Jur, V. D. Wheeler, D. J. Lichtenwalner, J. P. Maria, M. A. L. Johnson, Applied Physcis Letters 98 (2011) 042902.

[36] D. Bacorisen, R. Smith, J. A. Ball, R. W. Grimes, B. P. Uberuaga, K. E. Sickafus, W. T. Rankin, Nucl. Instrum. Methods Phys. Res., Sect. B 250 (2006) 36.

[37] M. R. Levy, R. W. Grimes, K. E. Sickafus, Phil. Mag. 84 (2004) 533.

[38] L. Minervini, R. W. Grimes, K. E. Sickafus, J. Am. Ceram. Soc. $83(2000) 1873$.

[39] B. P. Uberuaga, L. J. Vernon, Solid State Ionics 253 (2013) 18.

[40] C. Kinoshita, K. Hayashi, Adv. Ceram. 10 (1985) 490.

[41] K. Yasuda, C. Kinoshita, K. Fukuda, F. A. Garner, J. Nucl. Mater. 283-287 (2000) 937. 
1 [42] G. P. Wirtz, H. P. Takiar, J. Am. Ceram. Soc. 64 (1981) 748.

[43] P. Ágoston, K. Albe, Phys. Rev. B 81 (2010) 195205.

[44] B. C. H. Steele, J. M. Floyd, Proc. Brit. Ceramic Soc. 19 (1971) 55.

[45] M. Nolan, J. E. Fearon, G. W. Watson, Solid State Ionics 177 (2006) 3069

[46] D. A. Andersson, S. I. Simak, N. V. Skorodumova, I. A Abrikosov, B. Johansson, Proc. Natl. Acad. Sci. 103 (2006) 3518.

[47] A. F. Voter, Introduction to the kinetic monte carlo method, in: K. E. Sickafus, E. A. Kotomin, B. P. Uberuaga (Eds.), Radiation Effects in Solids, Springer, NATO Publishing Unit, Dordrecht, The Netherlands, 2006.

[48] P. J. Wilde, C. R. A. Catlow, Solid State Ionics 112 (1998) 173. 
${ }^{*}$ Graphical Abstracts_(for review)

Metal layer

Metal layer 